\title{
Prevalence of faecal incontinence in the community: a cross-sectional study in Singapore
}

\author{
Jason Wei-Min $\underline{\text { Lim }}^{1}$, MBBS, MRCS, Christian $\underline{\text { Heng }}^{2}$, MBBS, Mark Te-Ching Wong ${ }^{1}$, FRCSE, FAMS, \\ Choong-Leong Tang ${ }^{1}$, FRCSE, FAMS
}

INTRODUCTION Faecal incontinence $(\mathrm{FI})$ is a stigmatised condition that remains a silent affliction for many populations. To date, no local study has been performed to determine its prevalence in our population. The existing literature from the West has shown highly variable rates, ranging from $0.8 \%$ to $18.0 \%$. The aim of this study was to determine the crosssectional prevalence of $\mathrm{FI}$ in Singapore and to identify at-risk groups.

METHODS A door-to-door questionnaire survey was performed between February and March 2013. We randomly selected 1,000 individuals from the electoral roll to be surveyed using the validated Comprehensive Fecal Incontinence Questionnaire.

RESULTS A total of 381 participants agreed to be enrolled in the study. The mean age of the participants was 52 (range 21-86) years, and slightly more than half of the participants were female (52.8\%). Among the female participants, $73.1 \%$ had children ( $78.8 \%$ underwent normal vaginal delivery). The overall prevalence of $\mathrm{Fl}$ in our study population was $4.7 \%$. The prevalence of $\mathrm{FI}$ was observed to be significantly associated with increasing age $(p=0.004)$ and female gender $(p=0.029)$; women were three times more likely to suffer from FI than men.

CONCLUSION With the ageing population of Singapore, the results of the present study provide further impetus to continue public outreach efforts as well as develop clinical programmes that address the growing need for specialist treatments for people with $\mathrm{Fl}$.

Keywords: asian, faecal incontinence, prevalence, quality of life, questionnaire

\section{INTRODUCTION}

Faecal incontinence $(\mathrm{FI})$ is frequently referred to as a 'silent epidemic', explaining the inherent difficulty in obtaining reliable data that is reflective of the study population. $\mathrm{Fl}$ is a socially stigmatised condition that is extremely distressing to its sufferers, and a cause of both physical and psychological morbidity. While sufferers from all age groups have been reported, FI is known to predominantly affect the elderly. ${ }^{(1)}$ The predicament of this group of patients is worsened by the embarrassment associated with the condition and the lack of public education, both of which result in patients suffering in silence instead of seeking appropriate medical treatment.

Previous population studies have faced criticisms related to the variable definitions of $\mathrm{Fl}$, the use of non-validated assessment tools, poor response rates and nonrepresentative populations that are unable to reflect the true prevalence of $\mathrm{Fl}$ in the general population. ${ }^{(1-3)}$ These issues largely stem from a lack of consensus regarding the definition of $\mathrm{FI}$ and the appropriate assessment tools, as well as difficulties faced when trying to administer these assessment tools to the appropriate study population (given the sensitive nature of the condition). Previous studies have reported a wide range of prevalence rates of $\mathrm{FI}$ within the general population $(0.8 \%-18.0 \%)$. $^{(1-3)}$ Moreover, the inconsistent definition of $\mathrm{FI}$ - defined as the involuntary loss of control over the release of solid stools, liquid stools and/or gas - has accounted partly for the wide ranging prevalence rates in the aforementioned studies.
The primary objectives of the present study were to determine the prevalence of $\mathrm{FI}$ in the general Singapore population and to identify possible at-risk groups. It is our hope that this study, the first of its kind in Singapore, will give us valuable insight into this often neglected but significant condition in the local context, as well as enable healthcare administrators to more efficiently allocate the necessary resources to meet the needs of these vulnerable subgroups of patients.

\section{METHODS}

We identified a minimum sample size of 380 subjects for this study, based on a $10 \%$ estimated prevalence of $\mathrm{FI}$ in the general population and a 3\% (95\% confidence interval) margin of error. Via a computer-generated number allocation system, 1,000 subjects above the age of 21 years were randomly selected from the national electoral roll. In view of the prevailing data that women are at risk of developing $\mathrm{FI}^{(4,5)}$ we sampled 500 subjects from each gender (i.e. gender-stratified sampling was performed) to reduce sampling bias.

The primary component of the questionnaire used was the Comprehensive Fecal Incontinence Questionnaire (CFIQ), a validated and reliable assessment tool designed and tested in the general population of Auckland, New Zealand, by Macmillan et al. ${ }^{(6)}$ The CFIQ assesses both the severity of FI and its impact on quality of life. In addition, to assess whether the subjects perceived that they were suffering from a bowel control problem, the CFIQ included a single binary 'yes/no' question at the start of the questionnaire.

${ }^{1}$ Department of Colorectal Surgery, Singapore General Hospital, ${ }^{2}$ Yong Loo Lin School of Medicine, National University of Singapore, Singapore 
The Wexner Continence Grading Scale ${ }^{(7)}$ was adopted to define and grade the severity of FI in the present study, given the need for sensitivity in FI definition and a validated assessment tool for measuring severity of FI. In addition, we identified the Rockwood Fecal Incontinence Quality of Life (FIQOL) scale ${ }^{(4)}$ as a standardised assessment tool for evaluating the impact of FI on lifestyle in patients. A higher score on the Wexner Continence Grading Scale indicates a higher severity of FI (maximum: 20; minimum: 0 , indicating perfect continence), while a higher score on the FIQOL scale indicates a lower reduction in the patient's quality of life due to FI.

The validity of the data collected by various methods for stigmatised conditions such as $\mathrm{FI}$ has been criticised in previous studies. ${ }^{(8)}$ It has been suggested that for conditions that patients deem embarrassing, a self-administered, anonymous questionnaire would yield results of greater validity than face-toface interviews, as patients are expected to be less forthcoming with their answers when faced with an interviewer. ${ }^{(9)}$ However, we have not found this to be the case in our local Asian population. In a pilot study involving 1,000 participants who attended a public forum on FI in August 2012, we achieved a mere $10 \%$ response rate for the CFIQ, despite the application of a selected study population, anonymity, persistent data collection methods and simplified return of the CFIQ (in the form of postage-paid, self-addressed envelopes). We felt that the poor response could be due to a combination of factors, including the heterogeneity of our local population (in terms of spoken language and education level) and general public apathy.

Therefore, we opined that in our local context, it was more prudent for trained interviewers to administer the questionnaire via a face-to-face interview in the privacy of the participants' own homes. This method of data collection would allow the trained interviewers to: (a) personally reassure the participants of data confidentiality; (b) assist the participants in interpreting the questionnaire items; and (c) improve the participation rate by reducing the hassle of having to return the filled questionnaires. For this study, a field team of ten interviewers were trained to administer the questionnaire using a standardised technique, and deployed to perform house-to-house interviews with the 1,000 randomly selected subjects from February to March 2013.

The data collected from the present study was analysed using the Statistical Package for the Social Sciences version 17.0 (SPSS Inc, Chicago, IL, USA). Pearson's chi-square test and KruskalWallis test were applied, where appropriate, and all statistical tests performed were assessed at the conventional 0.05 level of significance.

\section{RESULTS}

Of the 1,000 subjects randomly selected and approached, $381(52.8 \%$ female, $47.2 \%$ male) agreed to participate in the present study, yielding a $38.1 \%$ response rate. The mean age of the participants was 52 (range 21-86) years. Among the female participants surveyed, $73.1 \%$ had previously given birth, with $78.8 \%$ having undergone a normal vaginal delivery (Table I).
Table I. Demographics and clinicopathologic characteristics of the study cohort $(n=381)$.

\begin{tabular}{lc}
\hline Parameter & No. (\%) \\
\hline Gender & $180(47.2)$ \\
Male & $201(52.8)$ \\
Female & $52(21-86)$ \\
Age group (yrs)* & \\
Child-bearing status & $147(73.1)$ \\
Given birth & $54(26.9)$ \\
Never given birth & \\
Mode of delivery & $103(70.1)$ \\
$\quad$ Normal & $31(21.1)$ \\
Caesarean section & $13(8.8)$ \\
Both & \\
\hline
\end{tabular}

*Data is presented as mean (interquartile range).

Table II. Fecal Incontinence Quality of Life ${ }^{(5)}$ scores of the study cohort $(n=381)$.

\begin{tabular}{lcc}
\hline Factor & $\begin{array}{c}\text { Mean score } \\
\text { (range) }\end{array}$ & $\begin{array}{c}\text { Maximum } \\
\text { score }\end{array}$ \\
\hline Lifestyle & $34.5(23-40)$ & 40 \\
Coping/behaviour & $22.5(11-36)$ & 36 \\
Depression/self-perception & $20.5(8-28)$ & 28 \\
Embarrassment & $7(4-12)$ & 12 \\
\hline
\end{tabular}

Based on the results of the present study, the overall prevalence of $\mathrm{Fl}$ in the local population was $4.7 \%$. Statistical analysis revealed a statistically significant association between $\mathrm{Fl}$ and female gender $(\mathrm{p}=0.029)$, with women being three times more likely to suffer from FI than men. A significant correlation between FI and increasing age was also noted ( $p=0.004)$. Participants aged $\geq 53$ years were found to have a fivefold increased probability of suffering from FI when compared with their younger counterparts (aged $<53$ years). FI was not found to be significantly associated with child-bearing status $(p=0.909)$ and normal vaginal delivery $(p=0.087)$, although the latter did show a trend toward significance.

The median score for Fl severity (measured using the Wexner Continence Grading Scale) was 5 (range 1-16). Analysis of the FIQOL scores revealed that FI sufferers scored poorly in the categories, coping/behaviour (22.5 out of 36) and embarrassment (7 out of 12) (Table II).

\section{DISCUSSION}

$\mathrm{FI}$ is a socially stigmatising condition, in which the afflicted frequently suffers in silence. This has, among other reasons, posed tremendous challenges on researchers and healthcare professionals alike, in their effort to determine the pervasiveness of the condition. The paucity of conclusive prevalence data within Asian populations, combined with the lack of public outreach programmes and established treatment protocols, has led to the undeserved neglect of FI sufferers.

The present study is the first cross-sectional population study on Fl in Singapore. Previous studies have been criticised for biased sampling of study populations due to the difficulty faced in recruiting patients for a study on a socially embarrassing 
condition. ${ }^{(10)}$ Studies that rely on convenience sampling (e.g. the use of hospitalised patients or patients attending medical reviews) tend to yield a higher prevalence rate, ${ }^{(3,11)}$ as it is likely that the recruited patients have medical conditions that could indirectly lead to a higher incidence of $\mathrm{Fl}$, and/or a greater awareness of their physical well-being when compared to healthy individuals. Therefore, it is not surprising that the local prevalence rate in our study (i.e. $4.7 \%$ ) is on the lower end of the spectrum when compared to other population studies. ${ }^{(2,3)}$ In fact, the prevalence rate obtained from the present study is lower than that noted in our pilot study (i.e. 8.8\%) involving participants attending a public forum on Fl in August 2012. This further illustrates the importance of not relying on convenience sampling when conducting studies on socially embarrassing conditions. Nonetheless, the prevalence rate obtained in the present study suggests a startling estimate of up to 200,000 people who might be suffering from this affliction.

The validity of data collection methods has been placed under scrutiny by many authors, particularly when the topic studied is a socially sensitive one. ${ }^{(10,12,13)}$ Dare et al suggested that, for socially stigmatising behaviours/conditions, the validity of data collected via face-to-face or telephone interviews would be lower than that of data collected via anonymous self-administered questionnaires. ${ }^{(13)}$ They postulated that patients are likely to feel embarrassed about the behaviours/conditions, and therefore have a greater likelihood of under-reporting the symptoms and other relevant information when faced with an interviewer. However, in our pilot study conducted on 1,000 participants at a public forum on Fl in August 2012, only 10\% of the participants returned their questionnaires, despite having been provided with selfadministered anonymous questionnaires (CFIQ), self-addressed postage-paid envelopes, and a dedicated telephone hotline and fast-tracked clinic appointment service for sufferers. These findings led us to postulate that, in the context of Singapore's multicultural population (with varying educational backgrounds, spoken languages and cultural beliefs), the use of a singlelanguage, self-administered questionnaire is possibly less effective in reaching out to the general population than private interviews. In particular, we noted that the elderly attending our colorectal clinics generally avoid sharing potentially embarrassing symptoms unless they have been reassured by a medical professional on the privacy of their conversation.

As such, our group adopted a face-to-face interview method of data collection, with interviews administered by a team of medical students who had been trained to administer the CFIQ. This method of data collection allowed us to reach the sample size required for the present study, as well as ensure that there were no 'non-response' items in the questionnaires, providing a high degree of fidelity within our collected data. The results of the present study support the assumption made regarding data collection, through the yield of much improved response rate. More importantly, the results offer an insight into the difference that exists between Asian and Western cultures in terms of how $\mathrm{Fl}$ is perceived.

The significant correlation between $\mathrm{Fl}$ and female gender is reaffirmed in the present study, which found women to be three times more likely to suffer from FI than men. While some studies have shown that factors such as intrapartum instrumentation, sphincter damage, fetal weight $>4 \mathrm{~kg}$ and prolonged second stage of labour are significantly associated with $\mathrm{FI}^{,}{ }^{(14-17)}$ conflicting data exists as to whether Caesarean delivery or childbearing status has any significant correlation with the incidence of FI. Lal et al found no significant difference in the incidence of $\mathrm{Fl}$ among a group of patients who delivered vaginally, a group who underwent elective Caesarean delivery and a group who underwent emergency Caesarean delivery. ${ }^{(18)}$ Nygaard et al, who extended the followup period of obstetric patients to 30 years after delivery, found that the incidences of flatus incontinence among women who underwent vaginal delivery and among women who underwent Caesarean delivery were similar (approximately 37\%). ${ }^{(19)}$ In the present study, we also found no significant association between FI and delivery status or mode of delivery. One possible explanation for this finding is that our local standards of perinatal and obstetric care likely minimises the extent of obstetric-related injuries during vaginal delivery. Furthermore, as Singapore is a small islandstate, expectant mothers have the advantage of quick access to a delivery-suite and are seldom subject to unnecessary prolonged labour, as compared to our Western counterparts.

Based on the results of the present study and reports in the prevailing literature, it can be surmised that advanced age and an often shorter anal canal length in the female gender are likely contributing factors to the incidence of FI. As we have identified the at-risk groups - females and individuals above the age of 52 years - efforts should be made to routinely screen these individuals for signs of $\mathrm{Fl}$. As a majority of patients would not volunteer such information due to embarrassment, it is the clinician's responsibility to actively engage them through appropriate questioning and a conscientious search for clues (e.g. stains on undergarments and/or perianal skin excoriation) that could identify a person who is silently suffering from Fl. It is our hope that the findings of the present study will encourage collaboration between the Ministry of Health and clinicians to broaden ongoing public outreach efforts, as well as to develop clinical programmes that will provide the much needed treatment to fight this 'silent epidemic'.

To conclude, the present study is the first to establish the prevalence rate of $\mathrm{FI}$ in Singapore. The $4.7 \% \mathrm{FI}$ prevalence rate, together with a significant correlation with increasing age and female gender, suggests that this socially distressing condition is likely to emerge as a significant health issue, which will accompany the impending 'silver tsunami' of our greying population. In a conservative Asian society where many patients choose to suffer in silence, clinicians have a duty to bridge the knowledge gap and shatter the stigma of embarrassment associated with $\mathrm{Fl}$, so as to provide the necessary treatment to optimise our patients' quality of life. The establishment of the Pelvic Floor Disorders Service in Singapore General Hospital $(\mathrm{SGH})$ is both timely and necessary to address this concern. SGH's public outreach efforts continue to raise public awareness of $\mathrm{Fl}$, and its state-of-the-art therapeutics seeks to offer the best patient outcomes for patients afflicted with FI. 


\section{REFERENCES}

1. Chassagne P, Landrin I, Neveu C, et al. Fecal incontinence in the institutionalized elderly: incidence, risk factors, and prognosis. Am J Med 1999; 106:185-90.

2. Nelson R, Norton N, Cautley E, Furner S. Community-based prevalence of anal incontinence. JAMA 1995; 274:559-61.

3. Giebel GD, Lefering R, Troidl H, Blöchl H. Prevalence of fecal incontinence: what can be expected? Int J Colorectal Dis 1998; 13:73-7.

4. Rockwood TH, Church JM, Fleshman JW, et al. Fecal Incontinence Quality of Life Scale: quality of life instrument for patients with fecal incontinence. Dis Colon Rectum 2000; 43:9-16.

5. Snooks SJ, Henry MM, Swash M. Faecal incontinence due to external anal sphincter division in childbirth is associated with damage to the innervation of the pelvic floor musculature: a double pathology. Br J Obstet Gynaecol 1985; 92:824-8.

6. Macmillan AK, Merrie AE, Marshall RJ, Parry BR. Design and validation of a comprehensive fecal incontinence questionnaire. Dis Colon Rectum 2008; 51:1502-22.

7. Jorge JM, Wexner SD. Etiology and management of fecal incontinence. Dis Colon Rectum 1993; 36:77-97.

8. Macmillan AK, Merrie AE, Marshall RJ, Parry BR. The prevalence of fecal incontinence in community-dwelling adults: a systemic review of the literature. Dis Colon Rectum 2004; 47:1341-9.
9. Tourangeau R, Yan T. Sensitive questions in surveys. Psychol Bull 2007; 133:859-83

10. Johanson JF, Lafferty J. Epidemiology of fecal incontinence: the silent affliction. Am J Gastroenterol 1996; 91:33-6.

11. Madoff RD, Parker SC, Varma MG, Lowry AC. Faecal incontinence in adults. Lancet 2004; 364:621-32.

12. Tourangeau R, Smith TW. Asking sensitive questions: the impact of data collection mode, question format, and question context. Public Opin Q 1996; 60:275-304.

13. Dare OO, Cleland JG. Reliability and validity of survey data on sexual behaviour. Health Transit Rev 1994; 4 Suppl:93-110.

14. Eason E, Labrecque M, Marcoux S, Mondor M. Anal incontinence after childbirth. CMAJ 2002; 166:326-30.

15. Gjessing H, Backe B, Sahlin Y. Third degree obstetric tears; outcome after primary repair. Acta Obstet Gynecol Scand 1998; 77:736-40.

16. de Leeuw JW, Struijk PC, Vierhout ME, Wallenburg HC. Risk factors for third degree perineal ruptures during delivery. BJOG 2001; 108:383-7.

17. Donnelly V, Fynes M, Campbell D, et al. Obstetric events leading to anal sphincter damage. Obstet Gynecol 1998; 92:955-61.

18. Lal M, H Mann C, Callender R, Radley S. Does cesarean delivery prevent anal incontinence? Obstet Gynecol 2003; 101:305-12.

19. Nygaard IE, Rao SS, Dawson JD. Anal incontinence after anal sphincter disruption: a 30-year retrospective cohort study. Obstet Gynecol 1997; 89:896-901. 